\title{
Artificial muscles based on Polyacrylonitrile based polymer electrolyte
}

\author{
Kumudu Perera ${ }^{1, *}$, K.P. Vidanapathirana ${ }^{1}$, M.A.K.L. Dissanayake ${ }^{2}$ \\ ${ }^{1}$ Department of Electronics, Wayamba University of Sri Lanka, Kuliyapitiya \\ ${ }^{2}$ Postgraduate Institute of Science, Peradeniya
}

\begin{abstract}
A system capable of converting chemical energy into mechanical energy can be treated as an artificial muscle or an actuator. Direct drive actuators incorporating materials like ferroelectric ceramics and piezoelectric polymers suffer from various limiting factors. It has been realized that polymers can be used to fabricate such muscles with appreciable performances. Movement of the muscles takes place as a result of incorporation and ejection of ions. In this study, the fabrication of a dry muscle using a Polyacrylonitrile (PAN) based electrolyte and two polypyrrole (PPy) electrodes and investigation of its performance is discussed.
\end{abstract}

The composition of the polymer electrolyte was 21mol\%PAN : 39mol\%EC : 33mol\%PC : $7 \mathrm{~mol} \% \mathrm{LiCF}_{3} \mathrm{SO}_{3}$ ) and it has a good mechanical stability. Two identical polypyrrole electrodes have been polymerized in the presence of dodecylbenzenesulfonate (DBS). An assembly of PPy : polymer electrolyte : PPy was formed in the normal atmosphere. Movements were observed using cyclic voltametry technique. Simultaneously, horizontal displacement of the muscle was observed. A rough estimation was done about the velocity of the muscles during oxidation and reduction. During reduction, a faster movement was obtained.

\section{INTRODUCTION}

Artificial Muscles or the actuators have the ability to convert chemical energy to mechanical energy. Almost all direct drive actuators exploit the energy conversion properties of transducing materials. But, none have shown a full response to the desired

\footnotetext{
* Corresponding author: kumudu@wyb.ac.lk
} 
specifications. They need high driving voltages and have exhibited small movements. With the exploration that conducting polymers can be used to make such muscles, some of those drawbacks could be overcome [1,2]. When they undergo reversible oxidation and reduction brought about by small electric potentials, incorporation and ejection of ions leads to contraction and expansion of the bulk polymer. Exchange of ionic species with the surrounding of polymer is achieved by placing the polymer in an electrolyte bath or in contact with a suitable electrolyte. While most studies have been carried out with liquid electrolytes, a considerable attention has been devoted lately to the realization of more efficient artificial muscles based on solid polymer electrolytes initiating an era of all solid polymer muscles [3]. Simply, an all polymer actuator resembles to a all polymer battery. The fabrication of dry muscles can be done by sandwiching a polymer electrolyte in between two polymer electrodes. In this study, our objective was to fabricate such muscle and observe its performance.

\section{EXPERIMENTAL}

Preparation of the electrolyte was done according to the hot pressed method [4]. Since the muscle was going to be used in the normal atmosphere, all the preparation steps were accomplished in the normal atmosphere. Deposition of PPy was performed on two different substrates; stainless steel and gold coated glass.

Electrochemical polymerization of pyrrole was done in the presence of sodium dodecylbenzesulfonate (SDBS) (ALDRICH)[4]. After polymerization, PPy films were peeled off from the stainless steel substrates with the use of a doctor blade. The films deposited on gold-coated glass could be peeled off easily with the gold backing. The configuration of the artificial muscles was PPy : DBS / PAN : EC : PC : $\mathrm{LiCF}_{3} \mathrm{SO}_{3} / \mathrm{PPy}$ : DBS.

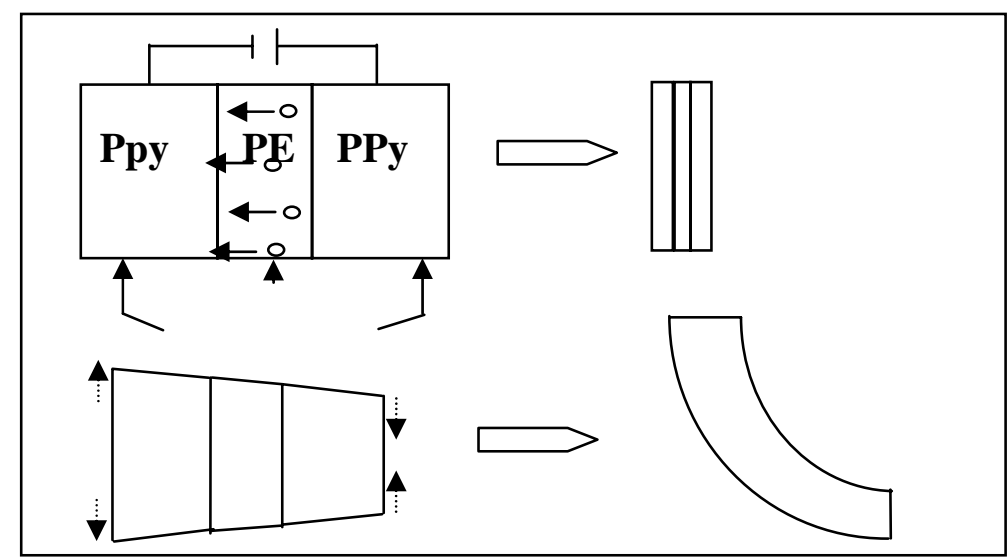

Fig. 1: Schematic of an artificial muscle (Top part illutrates the motion of ions occuring upon the application of a voltage. Bottom part corresponds to expansion and contraction due to insertion and deinsertion of ions which leads to motion of muscle) (PE : polymer electrolyte) 
In order to fabricate a muscle, two-rectangle shape PPy films were used with an identical size thin electrolyte film. One PPy strip was fixed on a well-cleaned glass slide and then the electrolyte film was put on it. The other PPy film was then placed on the electrolyte and pressed with another glass slide for better adhesion. Both sides of the assembly were heated at $140{ }^{0} \mathrm{C}$ for few seconds and left in the normal atmosphere for 1-2 days to form the sandwich. The assembly was of about $2 \mathrm{~cm}$ in height, $5 \mathrm{~mm}$ in width and $2.5 \mu \mathrm{m}$ in thickness. PPyPAN-PPy sandwich in Fig.1 was then connected to a potentiostat with the aid of micro SMD clip (RS radio component) as one PPy film to be the working electrode and the other to be both the reference and counter electrodes.

Deflections were observed while cycling between -2.0 to $2.0 \mathrm{~V}$. For this investigation, deflections were measured from fully reduced state $(-2.0 \mathrm{~V})$ to oxidized state $(2.0 \mathrm{~V})$ and then again to reduced state. In order to study the speed during oxidation and reduction, further measurements were taken for deflection of a muscle at different times. For this, first, a muscle was allowed for reduction by applying a voltage of $-3.0 \mathrm{~V}$. While it was moving, deflection was measured at different times. After $350 \mathrm{~s}$, the muscle was set for oxidation and readings for deflection were taken in the same way as above. Time period was limited to $350 \mathrm{~s}$.

\section{RESULTS AND DISCUSSION}

Movement of an artificial muscle fabricated with PPy : DBS / PAN / PPy : DBS within the potentail range -2.0 to $2.0 \mathrm{~V}$ is shown in Fig. 2.


Fig. 2: Motion of an artificial muscle of the configuration, PPy : DBS / PAN : EC : PC : $\mathrm{LiCF}_{3} \mathrm{SO}_{3} / \mathrm{PPy}$ : DBS (Potential range -2.0 - 2.0 V. Sweep rate $10 \mathrm{mV} / \mathrm{s}$. Thickness of PPy electrodes $10 \mu \mathrm{m}$, Thickness of electrolyte $50 \mu \mathrm{m}$, height and width of the assembly : $2 \mathrm{~cm}$ and $5 \mathrm{~mm}$ respectively)

The vertical positon was taken just after assembling. During cycling, one PPy film gets oxidized and reduced with reference to the other PPy film. The observed bending motion of the film assembly is associated with ion insertion and deinsertion. 
Muscles based on PPy : DBS had better movements when they were as free standing, ie. without gold backing. Although idea of using gold backing was to supply a continuous electronic path along the films, it resulted slow and sometimes no movements at all. Reason may be due to the marked rigidifying effect of gold.

Each time, it was required to perform few cyclings to initiate their movements. This may be possibly due to the fact that both PPy electrodes are in oxidized state at the beginning. To see ion movements, one PPy film should be in the oxidized state while the other is in the reduced state [5].

Working principle of artificial muscles is rather simple. When the ions are inserted into the polymer electrode, it causes an expansion and similarly a contraction upon deinsertion of ions. To permit the establishment of movements, ion insertion and deinsertion in two PPy films should occur simultaneously. For that, one PPy film should be at oxidized (or reduced) form while the other is at reduced (or oxidized) form. This well confirms the necessity of having two forms (oxidized and reduced) of PPy films in either sides. It has been reported that when PPy : DBS is cycled in non aqueous electrolytes, cations in the electrolyte move into the film during first reduction and make a neutral salt $\left(\mathrm{M}^{+} \mathrm{DBS}^{-}\right)$ [6].Thereafter, the film converts into an anion exchanging membrane. According to that, when we applied -2.0 V to the PPy film which is acting as the working electrode, $\mathrm{Li}+$ goes into the film and make LiDBS neutral salt. Then, during first oxidation which is followed by the first reduction, anions (in this case $\mathrm{CF}_{3} \mathrm{SO}_{3}{ }^{-}$) go into the membrane. In the second reduction, they are expelled out and this movement of inserting and de inserting of $\mathrm{CF}_{3} \mathrm{SO}_{3}{ }^{-}$repeats during next cycles. When the film serving as the working electrode undergoes reduction and oxidation consecutively, other film gets oxidized and reduced respectively. As a result, when one side gets contracted, other side gets extracted. Ultimate result is the bending of the muscle. Measurements of deflection while cycling is shown in Fig. 3.

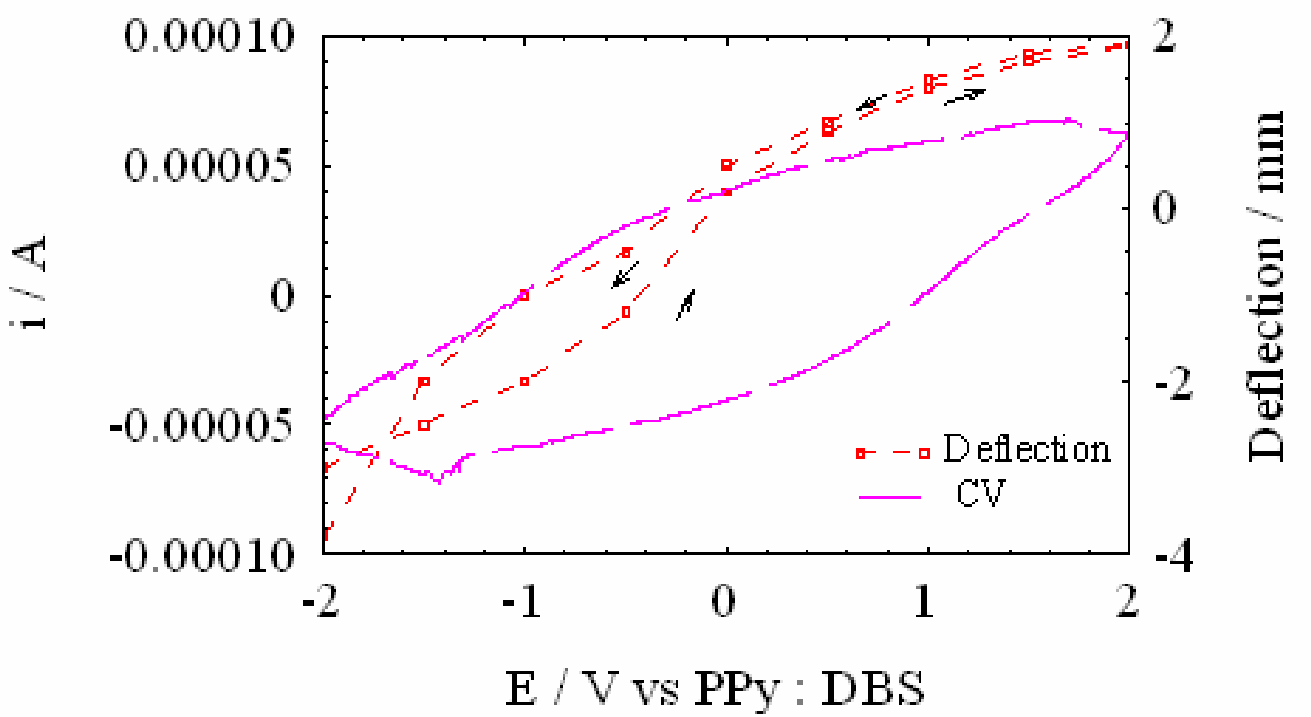

Fig.3 Measurements of deflection while cycling the artificial muscle (scan rate : $5 \mathrm{mV} / \mathrm{s}$ ) 
The vertical position has been taken as the zero deflection. Before measurements, muscle was set at reduced state and it moved $3 \mathrm{~mm}$. Slow movement occurred in oxidation cycle but, somewhat noticeable deflection was viewed during reduction. When the film gets reduced and oxidised, $\mathrm{CF}_{3} \mathrm{SO}_{3}{ }^{-}$are expelled out and absorbed in to the film. Due to the presence of the neutral salt, LiDBS, which is formed during first reduction, the absorption of anions is somewhat hindered. But, expelling (reduction) occurs well. Hence, the movement during reduction is higher than movements during oxidation. We experienced this all the time. The effect is pronounced in Fig. 4.

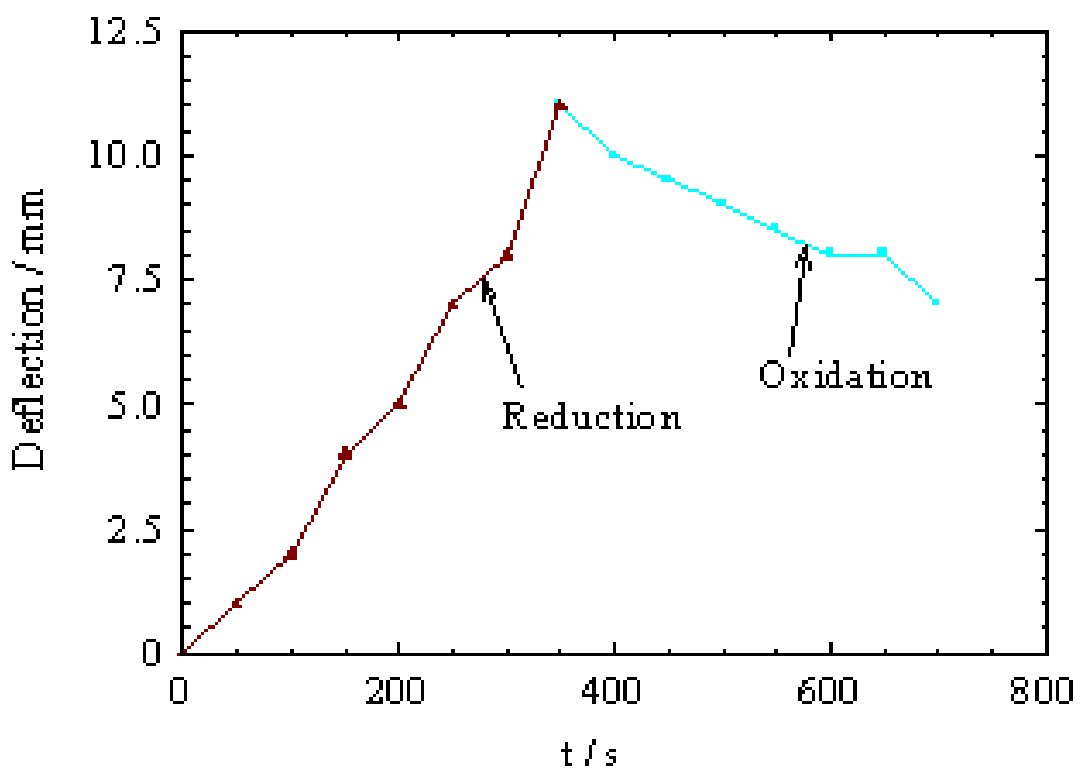

Fig. 4 Deflection while oxidation and reduction

In this case, we measured only the deflection of the free end of the muscle with time. Within $350 \mathrm{~s}$, it moved about $11 \mathrm{~mm}$ while getting reduced. But, during oxidation, its deflection was only $5 \mathrm{~mm}$ within the same time period. A rough estimation can be done to find the velocity of the muscle during reduction and oxidation. Values are approximately $0.03 \mathrm{~mm} / \mathrm{s}$ and $0.01 \mathrm{~mm} / \mathrm{s}$ for reduction and oxidation respectively. This well shows the faster movement during reduction.

On the other hand, ion movement has been reported to be accompanied with solvent transport in opposite direction [7]. That is solvent insertion is said to take place during reduction. It should be mentioned here that if solvent transport is dominant, an expansion can be expected during reduction. Contrarily, if ion transport is dominant, a contraction can be expected during reduction. According to our observation, which was a contraction during reduction, we claim that ion movement is dominating in the artificial muscles studied here.

Another important feature that could be envisioned is that the electrolyte composition used for batteries resulted poor adhesion and also low cycle lives when used for artificial 
muscles. It may be due to the more liquid condition and exudation of solvents in the electrolyte respectively. Therefore, the composition was alternated slightly to give the electrolyte a more brittle consistency. The composition is comparable to the one reported by T. W. Lewis et al [5]. It is given below in Table 1 .

Table 1: Composition of the electrolyte used to fabricate artificial muscles

\begin{tabular}{|c|c|c|c|c|}
\hline & PAN & EC & PC & $\mathrm{LiCF}_{3} \mathrm{SO}_{3}$ \\
\hline Wt. percentage & 12.63 & 37.89 & 37.89 & 11.59 \\
\hline Mol. percentage & 21.42 & 38.58 & 33.25 & 6.75 \\
\hline
\end{tabular}

\section{CONCLUSION}

Gel nature of the polymer electrolyte while retaining good mechanical stability assures proper adhesion between components. Nevertheless, the possibility of making them in very thin film form makes them more promising for the muscle application. It is seen that this electrolyte is a suitable candidate with respect to the application in artificial muscles.

\section{ACKNOWLEDGEMENT}

We wish to thank Prof. Steen Skaarup and Prof. Keld West at Technical University of Denmark for their assistance and support. We also acknowledge International Program for Physical Sciences (IPPS), Uppsala, Sweden for offering financial assistance by means of fellowships and equipments.

\section{REFERENCES}

1. T.F Otero, I Boyano, M.T Cortés and G Vázquez, Nucleation, non-stoiquiometry and sensing muscles from conducting polymers, Electrochimica Acta, 49(2004) 3719-3726

2. Dezhi Zhou, Geoffrey M. Spinks, Gordon G. Wallace, Churat Tiyapiboonchaiya, Douglas R. MacFarlane, Maria Forsyth and Jiazeng Sun, Solid state actuators based on polypyrrole and polymer-inionic liquid electrolytes, Electrochimica Acta, 48 (2003) 2355-2359

3. J.M. Sansiena, V. Olazabal, T.F. Otero, P. de Fonseca, C.N. De Paoli, M.A., Soft and wet conducting polymers for artificial muscles based on PPy and a solid polymer electrolyte working in air, J. Chem Soc., Chem. Communi., (1997) 2217-2225

4. Kumudu Perera, M.A.K.L. Dissanayake, P.W.S.K. Bandaranayake, S. Skaarup and K. West, Preparation of PAN : EC : PC : LiTf polymer electrolytes and characterization of Li / PAN : EC : PC : LiTf / PPy : DBS cells, Proceedings of the $7^{\text {th }}$ Asian Conference on Solid State Ionics, B.V.R. Chowdari and Wenji Wang , (2000), 483-489

5. T. W. Lewis, M. Pachetti, D. De Rossi, G. M. Spinks, G. G. Wallace, Development of an all polymer electrochemical actuator, Polymer Preprints, 38(2) (1997) 520-524 
6. Vidanapathirana, M.A. Careem, S. Skaarup and K. West, Electrochemical behaviour of a PPy(DBS) / polyacrylonitrile : $\mathrm{LiCF}_{3} \mathrm{SO}_{3}: \mathrm{EC}: \mathrm{PC} / \mathrm{Li}$ cell, Proceedings of the $10^{\text {th }}$ Asian Conference on Solid State Ionics, edited by B.V.R> Chowdari et al., (2206) 841-848

7. H. Lee, H. Yang, J. Kwak, Mass transport behaviour of PPy and PNMP films in acetonitrile solutions, Journal of Electroanalytical Chemistry, 468 (1999) 104-112 